\title{
La influencia del género en el aprendizaje matemático en España. Evidencias desde PISA
}

\author{
Gender Influence on Mathematics Achievement in Spain. \\ Evidence from PISA
}

Silvia Fuentes De Frutos, Víctor Renobell Santaren'

\section{Resumen}

Diferentes estudios muestran que la variable sexo incide en el rendimiento matemático del alumnado (Rodríguez, Delgado y Bakieva, 2011; Rodríguez-Mantilla, Fernández-Diaz y Jover, 2018). El presente trabajo indaga sobre la existencia y evolución de una brecha de género en el aprendizaje de las matemáticas en España, en base a los resultados de la evaluación PISA (Programme for International Student Assessment) en todas sus ediciones. Se valora también la competencia matemática del alumnado español sin diferencia de sexo. Se ha realizado una revisión bibliográfica de los último 30 años y un análisis multisectorial de los datos en base a los informes PISA. Los resultados del estudio indican una mejor competencia matemática en alumnos que en alumnas. Por otra parte, los resultados más actuales muestran una notable mejoría en el rendimiento matemático por parte del alumnado español en su conjunto..

\section{Palabras clave}

Educación, medida del rendimiento, matemáticas, diferencias por sexos.

\section{Abstract}

Different studies show that sex is a variable that affects mathematical performance (Rodríguez, Delgado and Bakieva, 2011; Rodríguez-Mantilla, Fernández-Diaz y Jover, 2018). This paper investigates the existence and evolution of a gender gap in mathematics achievement in Spain on the basis of the results of PISA (Programme for International Student Assessment) evaluation in all its editions. Mathematical competence of Spanish students is also analyzed without sex difference. A bibliographic review of the last 30 years and a multisectoral analysis of data based on PISA reports have been carried out. Results indicate better mathematical competence in boys. On the other hand, the most current results indicate a marked improvement in mathematical performance by Spanish students, in this case, without sex distinction.

\section{Keywords}

Education, achievemnt mesure, mathematics, sex differences.

\section{Cómo citar/Citation}

Fuentes De Frutos, Silvia; Renobell Santaren, Víctor (2020). La influencia del género en el aprendizaje matemático en España. Evidencias desde PISA. Revista de Sociología de la Educación-RASE, 13 (1), 63-80. http://dx.doi.org/10.7203/RASE.13.1.16042. 


\section{Introducción}

El objetivo del presente estudio es valorar si existe una brecha de género en el aprendizaje y rendimiento matemático (ambos conceptos se utilizan de manera indistinta) en España, y de ser así, en qué sentido ha evolucionado durante los últimos años. Se recurre al análisis multisectorial de los resultados en matemáticas de las alumnas y los alumnos españoles, en las todas las ediciones PISA (año 2000, año 2003, año 2006, año 2009, año 2012 y año 2015). Se realizará el análisis de los resultados con y sin distinción de sexo. Se parte de la hipótesis de la existencia de diferencias entre sexos en dicha prueba. La constatación de dicha diferencia es un dato relevante que contribuye al debate educativo sobre la cuestión aquí expuesta.

La metodología utilizada en el presente estudio es mixta. Por un lado, se ha utilizado un análisis bibliográfico, basado en varias bases de datos (WOS, SCOPUS, Google Académico y Dialnet), para identificar los artículos que tratan los factores explicativos de las diferencias en los resultados académicos en matemáticas. Se han seleccionado 84 fuentes bibliográficas, que datan desde 1986 hasta 2019, y que explican las diferencias entre sexos en relación al aprendizaje de las matemáticas. Se han descartado los artículos que no incidían en estas variables o que eran reiterativos con los mismos. Se han ordenado las propuestas, y se han extrapolado las principales conclusiones de los análisis que han realizado estos autores. Por otro lado, se han utilizado los datos facilitados por PISA y la OCDE (Organización para la Cooperación y el Desarrollo Económicos) desde el año 2000 para poder analizar las calificaciones y resultados obtenidos en España.

Enseñar matemáticas suele significar un trabajo complicado y difícil para los docentes en los diferentes niveles educativos. En este sentido, cabe destacar que han sido y son múltiples los esfuerzos realizados por la comunidad educativa para tratar de comprender los factores individuales, sociales y pedagógicos que inciden en esta problemática y conseguir acercar el mundo matemático a alumnos de todas las edades (Bishop, 2000; Zuaza y Rodríguez, 2002).

Son diversas las variables que parecen intervenir en el rendimiento matemático. Estudios e investigaciones apuntan a que estos factores, tanto biológicos como sociales, actúan de manera interrelacionada (Chamorro-Premuzic, Quiroga y Colom 2009; Chamorro-Premuzic y Furnham, 2008; Muelas, 2013).

Se ha puesto el acento en el papel de la inteligencia (Almeida, Guisande, Primi y Lemos, 2008; Bull y Scerif, 2001; Celis, 1986; Edel, 2003; Garbanzo y Guiselle, 2007; Markova y Powell, 1997; Muelas y Beltrán, 201; Pérez, González y Beltrán, 2009; Schillinger, Vogel, Diedrich y Grabner, 2018; Song et al., 2010) y el razonamiento matemático complejo (Desco et al., 2011) como variables que inciden de manera clara y notable en el rendimiento matemático. Se ha aludido a la importancia de factores familiares como el estilo parental (Pelegrina, García, y Casanova, 2002), el nivel educativo de los padres (Rodríguez-Mantilla et al., 2018) o a factores estructurales y sociológicos, como la inversión gubernamental en educación (Bolívar y López, 2009; Martínez, Reverte y Manzano, 2016), como variables que inciden en los resultados de los alumnos en matemáticas.

Desde otra perspectiva, se valoran también el autoconcepto y la autoestima (Marsh y Martin, 2010), la motivación (Blázquez, Álvarez, Bronfman y Espinosa, 2009; Rodríguez y Guzmán, 2018), la actitud hacia las matemáticas (Nasiriyan, Azar, Noruzy y Dalvand, 2011; Schaffer, 2000), y el papel de las emociones ante las matemáticas (Gil, Blanco y Guerrero, 2005; Gómez-Chacón, 2000; Gómez-Chacón, 2001; Song et al., 2010), como factores que influyen en el proceso de aprendizaje matemático (Dowker y Sarkar, 2016; Carey, Hill, Devine y Szücs, 2016; Schillinger et al., 2018). 
En este trabajo, el foco de atención se centra en el efecto del sexo en el aprendizaje y rendimiento matemático. Tal y como se ha descrito, la variable sexo es una de las variables con mayor capacidad de predicción del éxito en el rendimiento matemático (Farfán y Simon, 2017).

Se han observado diferencias entre sexos tanto en las actitudes ante las matemáticas como en el rendimiento en esta materia en diferentes estudios. Son diversos los estudios que demuestran que las alumnas suelen presentar actitudes más negativas y un rendimiento inferior. Dicha constatación se desprende como resultado del análisis de las respuestas al Inventario de Actitudes hacia las Matemáticas por parte de una muestra de 526 alumnas y alumnos de los ocho cursos de secundaria (González et al., 2012), o del estudio comparativo entre 181 estudiantes superdotados y 181 estudiantes no superdotados, en base a las respuestas dadas en diferentes pruebas y cuestionarios (Preckel, Götz, Pekrun y Kleine, 2008). Las diferencias se han evidenciado también en evaluaciones internacionales como PISA en sus diferentes ediciones. En más de la mitad de los países que pertenecen a la OCDE los alumnos obtienen mejores resultados que las alumnas en dicha evaluación. Además, existen diferencias los intereses y motivaciones relatados por alumnas y alumnos dentro del contexto de la misma evaluación (OCDE, 2010; Rodríguez-Mantilla et al., 2018; Ruiz de Miguel, 2009).

Por otra parte, también se ha sugerido que las alumnas tienden a mostrar una peor auto-percepción e imagen social en relación al desempeño matemático que los alumnos. Es lo que se refleja en un estudio longitudinal cualitativo en el que se analizaron los motivos que llevaron a dos alumnas superdotadas a elegir carreras universitarias sin contenido matemático (Lee y Sriraman, 2012). Las alumnas, además, se muestran menos seguras de ellas mismas en esta materia (Campos, 2006; Eudave, 1994; Frenzel et al., 2007; Morales, 1998; Ursini, 2010; Ursini, Ramírez y Sánchez, 2007; Ursini y Sánchez, 2008; Ursini, Sánchez, Orendain y Butto, 2004) y consideran menos placentero realizar ejercicios matemáticos. En sentido contrario, los alumnos demuestran menos ansiedad y desesperanza ante las tareas matemáticas que ellas. También se sienten más orgullosos y motivados tanto de forma intrínseca como extrínseca, según se constata a partir de los resultados de un metaanálisis realizado en base a las respuestas proporcionadas por 493.495 estudiantes de edades comprendidas entre los 14 y los 16 años (Else-Quest, Hyde y Linn, 2010).

Se observa un patrón relacionado con la edad. Bethencourt y Torres (1987) llevaron a cabo un estudio a partir de una muestra de 1078 estudiantes que cursaban diferentes cursos de educación primaria a los que se les administró una serie de problemas aritméticos verbales. Las niñas mostraron un mejor rendimiento hasta los 8 años, pero a partir de esas edad, los niños establecieron su superioridad en esta materia. Cabe añadir que la visualización espacial, el pensamiento numérico y la resolución de problemas son las áreas más afectadas. El inicio de la adolescencia parece ser el periodo de crecimiento en el que las diferencias entre sexos, en rendimiento matemático, toman mayor relevancia (Farfán y Simón, 2017).

Según se desprende del análisis bibliográfico realizado en el presente trabajo, la diferencia en el desempeño matemático entre sexos es un fenómeno que puede ser abordado desde diversas perspectivas (Del Río, Strasser y Susperreguy, 2016; Ursini, 2013). En el presente estudio, el enfoque se centra en el análisis de los factores sociales que inciden en el rendimiento matemático y en su repercusión en la esfera individual, enfoque que siguen la mayoría de los trabajos del ámbito de análisis y sus principales referentes.

Se ha subrayado que la diferencia significativa en el rendimiento matemático entre sexos estaría, en parte, relacionada con las políticas y prácticas educativas (Consorcio PISA, 2004). En este sentido, aspec- 
tos como la interacción establecida entre profesor y alumno, las estrategias elaboradas por el profesor, con el objeto de propiciar la equidad de sexos, y las expectativas sobre el aprendizaje de las matemáticas, en función del sexo del alumnado, parecen poseer una gran relevancia en el aprendizaje matemático del alumnado (Hanna 2003; Ramírez 2006).

Los profesores tienden a asociar el mejor desempeño en matemáticas con el sexo masculino (Hanna, 2003; Ursini, 2009 y 2010; Ursini y Sánchez, 2008). Se ha llegado incluso a describir que los alumnos reciben más atención y mayor calidad de instrucción que las alumnas (Bueno, 2006; Lee y Sriraman, 2010; Mingo, 2006). Esta constatación situaría a los centros educativos como propulsores en la creación de estereotipos de género en relación al desempeño matemático (Ursini, Ramírez- Mercado, 2017).

También la familia es un factor clave en la asunción del conocimiento acerca de los roles y estatus que poseen alumnos y alumnas en la sociedad. Otros dos agentes socializadores muy importantes, los medios de comunicación y el entorno social, inciden también en todo lo que se cree y espera sobre o un hombre o una mujer en nuestra sociedad, ya sea a nivel individual o social (Blázquez et al., 2009; Coyne, Linder, Rasmussen, Nelson y Collier, 2014; Del Río et al., 2016; García, 2204; Ursini, Ramírez 2017).

Los estereotipos de género, en relación a la poca capacidad de las alumnas para las matemáticas, poseen una fuerte influencia en el proceso de aprendizaje matemático (Gunderson, Ramírez, Levine y Beilock, 2011; Herbert y Stipek, 2005; Kurtz-Costes, Rowley, Harris-Britt y Woods, 2008; Lee y Srirman, 2012; Mizala, Martínez y Martínez, 2015; Ursini 2009; Ursini y Sánchez, 2008). Se trata de un estereotipo de género ampliamente extendido en el mundo (Nosek, Smyth, Sriram y Lindner, 2009).

Los mismos alumnos parecen influir en su rendimiento matemático a través de sus propios estereotipos. Se ha apuntado hacia el hecho de que el alumnado sostiene estereotipos sobre diferencias en el rendimiento escolar en función del sexo desde una temprana edad, adjudicando una mejor competencia matemática a los niños que a las niñas. Fue ese el resultado de una investigación en la que participaron 180 niños de educación infantil a los que se les aplicaron medidas de estereotipos explícitos e implícitos (Del Río et al., 2016).

Se ha señalado también que los propios alumnos recurren de manera más frecuentes a los padres y a los familiares de sexo masculino que a madres y a familiares de sexo femenino cuando requieren ayuda a la hora de realizar las tareas matemáticas (Ursini, 2009).

Todos estos aspectos parecen tener repercusión negativa sobre el propio autoconcepto y autoestima de las alumnas y en sus emociones hacia las matemáticas, que incluyen niveles más elevados de ansiedad en comparación con los alumnos. En contraposición, los alumnos muestran un mayor nivel de autoeficacia y más confianza en ellos mismos para solucionar las tareas matemáticas. Cabe subrayar que las competencias desarrolladas por las alumnas durante los años previos a la escolarización podrían influir en las diferencias constatadas (Brandell y Staberg, 2008; Inda-Caro, Rodríguez-Menéndez y Peña-Calvo, 2010; Rodriguez-Mantilla et al., 2018).

Sin embargo, no todos los estudios existentes hasta la fecha apuntan hacia la dirección aquí citada (González-Pienda et al., 2012; Kloosterman, Tassell, Ponniah, y Essex, 2001). Forgasz (2000) realizó una revisión extensa en estudiantes australianos, en base a la aplicación de dos escalas de actitudes (Mathematics as a gendered domain y Who and mathematics) hacia las matemáticas, y sus datos revelaron que los alumnos consideran las matemáticas más difíciles que las alumnas, precisando más ayuda adicional. 
Además, en este caso, las alumnas se interesaban y valoraban más positivamente las matemáticas que los alumnos.

Recientemente, en el estudio llevado a cabo por Gasco-Taxabarri (2017), se describe que las alumnas tienen tendencia a ordenar y a gestionar mejor los conocimientos matemáticos y que tienden a pedir ayuda, en caso de duda, con más facilidad que los alumnos. También que ambos sexos utilizan de manera similar la repetición, elaboración, planificación y el seguimiento-regulación como estrategias de aprendizaje para las matemáticas, así como el entorno de estudio. Este trabajo contó con una muestra de 565 estudiantes de $2 .^{\circ}, 3 .^{\circ}$ y $4^{\circ}$ curso de educación secundaria a los que se les administró una adaptación del cuestionario Motivated Strategies for Learning Questionnaire.

Por otra parte, actualmente, el rendimiento a gran escala en el área matemática se evalúa a través de diversas encuestas internacionales. PISA es un estudio de la OCDE que mide los conocimientos y destrezas de alumnos de 15 años en Lectura, Matemáticas y Ciencias, además de en un área de innovación (en la edición de 2015) en la que se evalúa la competencia en resolución de problemas colaborativos.

La evaluación PISA incluye a los países de la OCDE y a un número variable de países no miembros, llamados «asociados», y se lleva a cabo cada 3 años. El indicador que suele recibir más atención es el lugar ocupado por cada país según la puntuación media en las pruebas de rendimiento académico. De hecho, se puede afirmar que la puntuación de los países en estas clasificaciones afecta a las medidas educativas tomadas desde las diferentes instituciones (Takayama, 2008).

PISA se ha constituido en un medio, de prestigio internacional, para conocer las diferencias académicas existentes entre países y, en ocasiones, también dentro de un mismo país. Además, puede concebirse como un indicador del resultado de las políticas educativas implementadas en los diferentes territorios participantes en el estudio, así como una herramienta para dilucidar qué estrategias educativas parecen funcionar mejor. Cabe destacar, sin embargo, que es necesario tratar los resultados con cautela y precaución, debido a que son muchas las variables y los factores ajenos al ámbito de la política educativa los que influyen en los resultados. Entre estos, se ha destacado que no se trata de una prueba adaptada a las necesidades económicas y sociales de cada país (Cadenas y Huhertas, 2013).

PISA no tiene en cuenta que en diferentes países es importante potenciar diferentes competencias, por ejemplo, los conocimientos agrícolas en un país centrado esencialmente en esta actividad. Por otro lado, es cuestionable la selección de materias valoradas en el informe PISA. A pesar de analizar en profundidad tres materias muy relevantes, prescinde de otros conocimientos y habilidades esenciales para el desarrollo de los alumnos (Cadenas y Huhertas, 2013). Otro aspecto negativo sería el hecho de que se generen presiones por querer igualar a los países con rendimiento académico más alto y que, en consecuencia, se adopten prácticas educativas de estos países que pueden no ser extrapolables por diferentes razones (Takayama, 2008).

\section{Situación actual de las matemáticas en España. Datos de PISA}

En este apartado se revisan los resultados españoles en PISA desde el año 2000 hasta la edición del año 2015, la última publicada. En la Tabla I se exponen las medias de las puntuaciones obtenidas por los alumnos, sin y con distinción de sexo, en las seis ediciones. En global, sin distinción de sexo, se observa que el alumnado español han mejorado su rendimiento matemático en un 2,06\% desde el año 2000 (primera edición) hasta 2015 (última edición analizada). Si diferenciamos por sexos, observamos que, en el 
caso de las alumnas, la diferencia en estos 15 años es de un 2,30\%. La diferencia en el caso de los alumnos es de un 1,82\%. Tal como puede observarse, la tasa de mejora de las niñas es mayor (casi medio punto más) que la de los niños.

Tabla I. Media de resultados de PISA en España y por sexo

\begin{tabular}{ccccccc}
\hline PISA & 2000 & 2003 & 2003 & 2003 & 2003 & 2003 \\
\hline España (media) & 476 & 485 & 480 & 483 & 484 & 486 \\
\hline Alumnas & 467 & 481 & 476 & 473 & 476 & 478 \\
\hline Alumnos & 485 & 490 & 485 & 492 & 492 & 494 \\
\hline
\end{tabular}

La diferencia observada en las diferentes ediciones resulta significativa $(p=0,005)$.

Fuente: Ministerio de Educación, Cultura y Deporte (2001, 2004, 2005, 2014, 2016).

\section{Gráfico 1. Mejoras en rendimiento matemático por sexos}

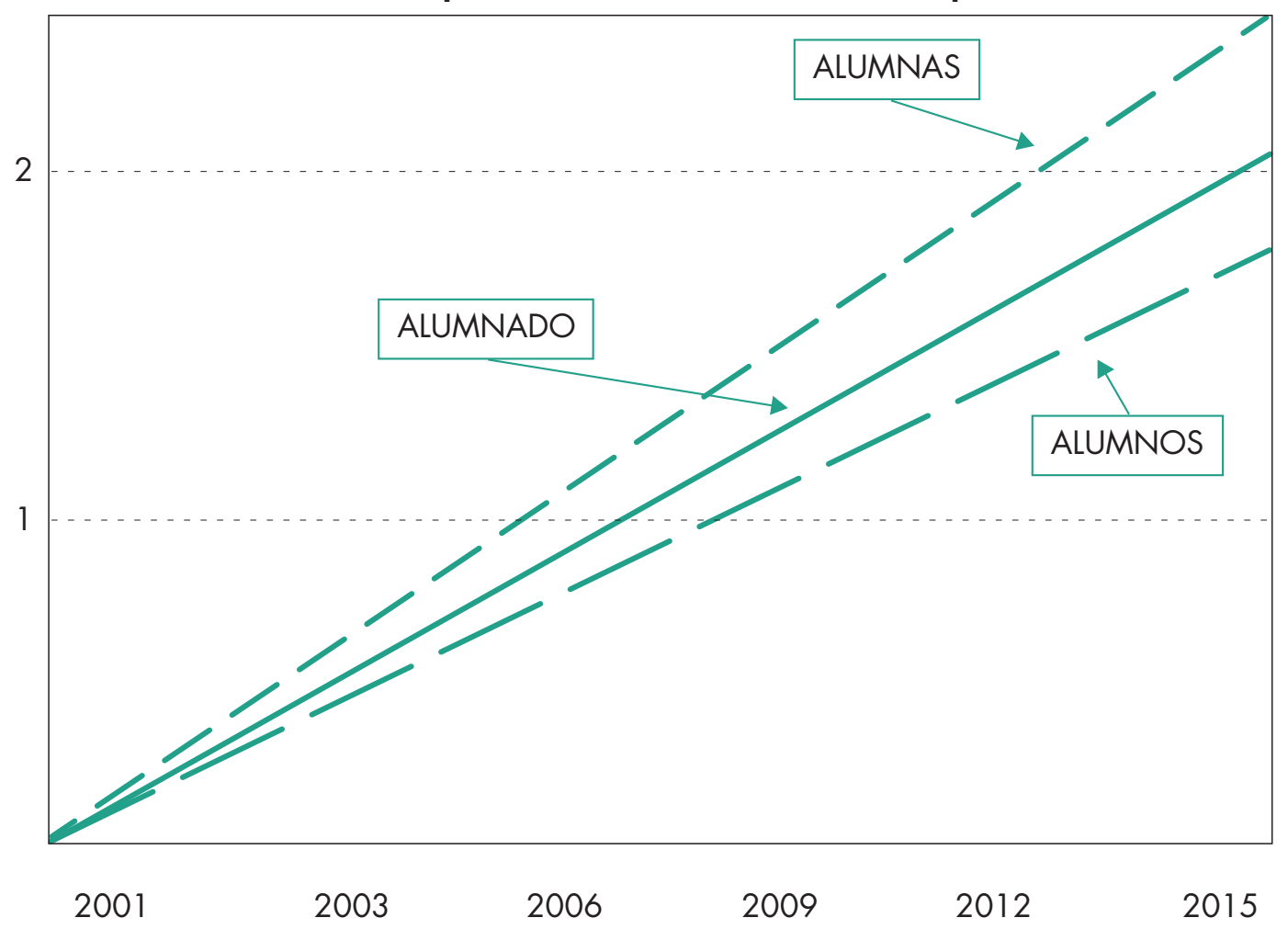

Evolución proyectada en el rendimiento medio del alumnado en España.

Fuente: Ministerio de Educación, Cultura y Deporte (2001, 2004, 2005, 2014, 2016).

\subsection{PISA 2000}

En PISA 2000 participaron 32 países, entre ellos España. En esta edición de PISA, se observa un claro efecto de la variable sexo en el rendimiento matemático, al presentar las alumnas españolas una diferencia de 18 puntos en sentido negativo (un 3,71\%) respecto a los alumnos. En la mayoría de los países participantes, las diferencias de sexos se producen en la misma dirección que las observadas en España, pero en diferente proporción.

Los resultados del alumnado español (aquí sin distinción de sexo) muestran una diferencia importante (24 puntos por debajo) en matemáticas (476 puntos) respecto a la media (500 puntos). Lo que significa un $4,8 \%$ de diferencia negativa respecto a la media. 
Un apunte positivo sería que España mejora la desviación típica del conjunto de países de la OCDE (100) en 9 puntos. A partir de dicho dato, se puede hipotetizar que se está ofreciendo equidad e igualdad en las oportunidades proporcionadas al alumnado español.

\subsection{PISA 2003}

En la edición de 2003 participaron 41 países y en España se analizaron por separado las competencias de Castilla y León, País Vasco y Cataluña. En su conjunto, las alumnas españolas obtienen en matemáticas una puntuación media (481 puntos) menor que la de los alumnos (490 puntos). La diferencia de 9 puntos (un 1,84\%) a favor de los alumnos es estadísticamente significativa. Destaca la dispersión entre las diferencias obtenidas en el País Vasco (1 punto) y en Cataluña (18 puntos). El sentido de las diferencias entre las alumnas y los alumnos españoles es el mismo que en el promedio de países de la OCDE y que en el de la mayoría de los países, con la excepción de Islandia y Tailandia.

Sin contemplar la distinción por sexos, los resultados del alumnado español en matemáticas siguen por debajo del promedio de la OCDE (485 frente a 500), siendo esta diferencia estadísticamente significativa. Castilla y León obtiene un promedio de 503 puntos, el País Vasco de 502 y Cataluña de 494 puntos.

De nuevo, España sigue posicionándose bien respecto a la equidad de los resultados, tal y como muestra su escasa dispersión.

\subsection{PISA 2006}

En la edición de 2006 participaron 57 países. En España se analizaron por separado las competencias de Castilla y León, País Vasco, Cataluña, Galicia, Asturias, Cantabria, La Rioja, Aragón, Andalucía y Navarra. En competencia matemática, la media española vuelve a situar 9 puntos (un 1,84\%) por encima a los alumnos respecto a las alumnas, se trata de una diferencia similar al promedio de la OCDE (11 puntos). En todas las Comunidades Autónomas analizadas, las diferencias en matemáticas son también favorables a los alumnos, en valores superiores a la media española, salvo en el País Vasco. Destacar las diferencias a favor del alumnado en Aragón, donde éstas se sitúan en 18 puntos.

La media del alumnado español (en global), en matemáticas, desciende 5 puntos respecto a la media de 2003 y se sitúa en 480 puntos, las diferencias entre las 3 ediciones se revelan, sin embargo, ligeras. La media de los países restantes se sitúa en 498 puntos.

Sin distinción de sexos, la media de los resultados en La Rioja es 526 puntos, la de Castilla y León y Navarra 515 puntos, la de Aragón 513 puntos, la de Cantabria 502 puntos, la del País Vasco 501 puntos, la de Asturias 497 puntos, la de Galicia 494 puntos, la de Cataluña 488 puntos y, finalmente, la media andaluza se sitúa en 463 puntos. Son, por lo tanto, diferencias abultadas que llegan a diferenciarse en 63 puntos. En esta ocasión los resultados peores del alumnado español (sin distinción de sexos) demuestran que el repunte positivo de los años pasados no es una tendencia firme.

La equidad en los resultados vuelve a mostrarse como un punto fuerte del sistema educativo español, se trata de un nivel próximo al de los países nórdicos. 


\subsection{PISA 2009}

En 2009 se analizaron 65 países y en España se analizaron las competencias, por separado, de Castilla y León, País Vasco, Cataluña, Galicia, Asturias, Cantabria, La Rioja, Aragón, Andalucía, Navarra Baleares, Canarias, Madrid, Murcia y las ciudades autónomas de Ceuta y Melilla. El promedio de los alumnos en matemáticas vuelve a ser más elevado que el de las alumnas, en esta edición la diferencia se sitúa en 19 puntos (un 3,86\%), cifra superior a la de la OCDE (11 puntos). Se trata de la mayor diferencia entre alumnos y alumnas detectada.

El promedio de España en matemáticas (sin distinción de sexos) se sitúa en 483 puntos. Los resultados españoles globales en competencia matemática en 2009 son muy similares a los de ejercicios anteriores y se sitúan por debajo de la media de la OCDE (496). Las comunidades autónomas de País Vasco y Navarra cuentan con un $13 \%$ de alumnos con alto nivel de rendimiento matemático, porcentaje coincidente con el promedio de la OCDE. Con cifras ligeramente superiores a la del promedio OCDE se sitúan Castilla y León (15\%), Aragón (14\%) y La Rioja (15\%). Cataluña, Madrid, Asturias y Cantabria cuentan con un $11 \%$ del alumnado con alto nivel de rendimiento matemático. Murcia y el promedio español se sitúan en el 8\% y, en Galicia, el 7\% del alumnado presenta un rendimiento matemático alto. El resto de las comunidades autónomas tienen un 4\% del alumnado o menos en estos niveles altos de rendimiento. De nuevo, los resultados entre las diferentes Comunidades Autónomas se muestran poco homogéneos.

Los resultados obtenidos en esta edición vuelven a corroborar que el sistema educativo español presenta un nivel de equidad y homogeneidad notable.

\subsection{PISA 2012}

En PISA 2012 participaron 65 países y en España se analizaron por separado los resultados de Andalucía, Aragón, Principado de Asturias, Illes Balears, Cantabria, Castilla y León, Cataluña, Extremadura, Galicia, La Rioja, Madrid, Región de Murcia, C. Foral de Navarra y País Vasco. En matemáticas, a nivel estatal, los alumnos obtienen puntuaciones más altas (492 puntos) que las alumnas (476 puntos). Los alumnos obtienen así unas puntuaciones un 3,25\% superiores a la de las alumnas. Este porcentaje es mayor que el promedio de los países de la OCDE, pero inferior al de Japón y Corea del Sur, países que ostentan las puntuaciones más altas en esta prueba. En relación con las Comunidades Autónomas participantes en el estudio, las diferencias en los resultados en función del sexo del alumnado son heterogéneas, con la mayor diferencia en Cataluña (+22 puntos) y la menor en Galicia ( +2 puntos).

España, sin distinción de sexo, consigue una puntuación de 484 puntos en matemáticas, 10 puntos inferior al promedio de la OCDE (494 puntos) y 5 puntos más baja del promedio de la UE (489 puntos). No se producen cambios estadísticos significativos con relación a las ediciones pasadas. En relación con las diferentes Comunidades Autónomas participantes, las puntuaciones más altas en matemáticas corresponden a Comunidad Foral de Navarra (517 puntos), Castilla y León (509 puntos), País Vasco (505 puntos), la Comunidad de Madrid (504 puntos) y La Rioja (503 puntos), siendo significativamente superiores al promedio de la OCDE. Las puntuaciones de Extremadura (461 puntos), Murcia (462 puntos) y Andalucía (472) son las más bajas dentro del contexto español y reflejan, de nuevo, una importante heterogeneidad en los resultados entre Comunidades Autónomas. 
Respecto a la equidad, cabe destacar que el sistema educativo español sigue siendo equitativo, a niveles comparables a los de los países nórdicos, tal y como se había constatado anteriormente.

\subsection{PISA 2015}

En la edición de 2015, edición con los últimos datos publicados en el momento de realización del presente trabajo, participaron 72 países. En España se analizaron por separado las competencias de todas las comunidades autónomas. En matemáticas, sigue observándose un promedio más elevado en el caso de los alumnos respecto a las alumnas, tal y como viene siendo la tendencia desde la edición del año 2000. En esta ocasión, la diferencia se sitúa en 16 puntos (3,24\%). La diferencia entre los resultados de alumnos y alumnas llega a 8 puntos, en el promedio de los países de la OCDE, y a 11 puntos en el total UE, siendo los alumnos quienes obtienen mejores resultados. Únicamente en Finlandia las alumnas consiguen resultados significativamente superiores.

En lo que se refiere a las Comunidades Autónomas, las mayores diferencias en el rendimiento en matemáticas entre alumnos y alumnas se constatan en la Comunidad de Madrid y Cataluña, más de 18 puntos en ambos casos, y las más bajas se observan en Castilla y León con 5 puntos a favor de los alumnos. Tampoco son significativas las diferencias en la Región de Murcia, Extremadura, Islas Baleares y Aragón. España obtiene una puntuación en matemáticas (aquí sin distinción de sexo) de 486 puntos, 4 menos que el promedio de la OCDE (490) y 7 puntos por debajo del total de la UE (493). Los resultados de España son similares a los de Portugal (492), Italia (490), Islandia (488), Luxemburgo (486) y Letonia (482). En cuanto a las Comunidades Autónomas españolas, las puntuaciones más altas en matemáticas corresponden a la Comunidad Foral de Navarra (518 puntos), Castilla y León (506 puntos), La Rioja (505 puntos) y la Comunidad de Madrid (503 puntos), sus puntuaciones son significativamente superiores al promedio de la OCDE (490 puntos). Por otro lado, Murcia (470 puntos), Andalucía (466 puntos) y Canarias (452 puntos) presentan las puntuaciones más bajas. Vuelve, por lo tanto, a ser evidente la heterogeneidad de los resultados españoles en función de la Comunidad Autónoma.

España sigue situándose entre los países más equitativos. El impacto del índice social, económico y cultural se cifra netamente por debajo del impacto en el conjunto de países OCDE.

Un análisis de todos los datos aquí expuestos permite señalar la existencia de una brecha de género en el rendimiento matemático, a favor de los alumnos, tanto en España como en la gran mayoría de los países participantes. Tal y como se puede observar, este fenómeno se repite en las diferentes ediciones del estudio y persiste cuando se analizan los resultados de las Comunidades Autónomas por separado. Lejos de reducirse, la diferencia en el rendimiento matemático por sexos, en base a los resultados más actuales, se muestra estable e incluso superior respecto ediciones pasadas (2003 y 2006). Se trata de una cuestión relevante y preocupante que debería suscitar la implementación de medidas por parte de las instituciones educativas y un mayor esfuerzo en las investigación para aclarar los condicionantes de dicho desfase.

Respecto a los datos globales, puede constatarse una clara mejoría en las competencias matemáticas del alumnado español en la última edición respecto a las ediciones previas. Destacar, sin embargo, la heterogeneidad en los resultados hallada a través del análisis por Comunidades Autónomas. Se trata de una mejora que acerca la media española a la de la OCDE. En este contexto, supone el mejor dato desde el comienzo de la evaluación PISA. 


\section{Conclusiones}

En relación a los objetivos planteados en el presente estudio, subrayar que el análisis de las ediciones PISA demuestra la existencia de una brecha de género en el rendimiento matemático en España. En consonancia con lo observado en la mayor parte de los países analizados a través de las seis ediciones PISA, los alumnos españoles presentan mejores puntuaciones en matemáticas que las alumnas españolas en porcentajes que varían en función de la edición. El análisis efectuado por Comunidades Autónomas revela que dicha tendencia se repite en todas ellas a lo largo de las diferentes ediciones. Tal y como se ha constatado, la brecha de género detectada resulta estable e incluso acrecentada durante las últimas ediciones respecto a ediciones anteriores.

Por otra parte, los datos más actuales revelan una mejora en el rendimiento matemático en el alumnado español (sin distinción de sexo) en relación a ediciones previas. Se trata de una mejora que acerca la media española a la de la OCDE. En este contexto, este supone el mejor dato desde el comienzo de la evaluación PISA. Cabe destacar, como contrapunto, la heterogeneidad en los resultados observada al analizar los datos por Comunidades Autónomas.

Si bien a nivel individual el rendimiento matemático parece estar influido por factores en parte biológicos, como la inteligencia y determinados rasgos de la personalidad, las aportaciones bibliográficas, expuestas en el presente trabajo, permiten poner el acento en los factores contextuales para explicar las diferencias de sexo en el rendimiento matemático. Entre estos cabe destacar la importancia de los estereotipos y la temprana asunción de ellos por parte de las niñas, así como la existencia de limitaciones socioculturales.

La decisión de elegir o declinar una carrera con contenido matemático en los estudios superiores, podría verse afectada por dichos factores, eliminando así la posibilidad para ciertas mujeres de acceder a trabajos competitivos (OECD, 2007; 2010). De hecho, las matemáticas se han constituido socialmente como una herramienta de selección y segregación intelectual en el ámbito académico y profesional (Cantoral, Montiel y Reyes, 2014; Llanos y Otero, 2015; Valero et al., 2013).

Concretamente, en el caso español, se ha constatado que el porcentaje de alumnas en las carreras tecnológicas lleva estancado desde los años 90. El porcentaje de alumnas en las carreras de Ingenierías, Ciencias Experimentales y Arquitectura es de un 30\%, en Informática la cifra cae por debajo del 15\% (Instituto de la Mujer y para la Igualdad de Oportunidades, Ministerio de la Presidencia, Relaciones con las Cortes e Igualdad).

Una de las metas de nuestro sistema educativo debería ser el logro de la equidad de género. Para ello será necesarios propiciar cambios sociales importantes y hacer efectivas políticas adecuadas centradas, entre otros aspectos, en favorecer los estudios científicos y matemáticos para el público femenino. Habrá que tener en cuenta, sin embargo, que las condiciones sociales y la propia construcción del género en la sociedad actual hacen de freno ante este nuevo reto educativo.

Deconstruir el género socialmente para poder dar más opciones igualitarias y disminuir así la brecha discriminatoria entre el alumnado resulta también una medida a tener en cuenta. Para ello será necesario profundizar en la comprensión del autoconcepto interno e ir más allá de las propias construcciones sociales. En la misma línea, son diversos autores que opinan que el camino a seguir para mejorar e integrar las matemáticas en el quehacer del alumnado femenino está en la nueva construcción del género y en el cambio social de los modelos e ideales a seguir (Calvo, 2018). 
Por otro lado, queda por comprobar si la mejora observada en el rendimiento matemático en el alumnado español, en su conjunto, se trata de una tendencia al alza. Asimismo, queda también por valorar el conjunto de variables y factores, tanto sociales como individuales, que han ayudado a que se haya producido dicha mejora en el rendimiento matemático.

Resultan muy interesantes y motivadores los datos en relación a la homogeneidad de los resultados obtenidos en el caso español, índice que demuestra igualdad de oportunidades para el alumnado, independientemente de la posición económica y social. El hecho de que nuestro país esté bien posicionado en base a este indicador invita a seguir trabajando en la misma línea seguida hasta ahora a nivel socioeconómico y cultural.

\section{Referencias bibliográficas}

Almeida, Leandro S.; Guisande, M. Adelina; Primi, Ricardo y Lemos, Gina Cláudia (2008): "Contribuciones del factor general y de los factores específicos en la relación entre inteligencia y rendimiento escolar". European Journal of Education and Psychology, 1 (3), 5-16. DOI: https://doi.org/10.30552/ejep. v1i3.13.

Bethencourt, José Tomás y Torres, Esteban (1987): “La diferencia de sexo en la resolución de problemas aritméticos: un estudio transversal”. Infancia y aprendizaje, 38, 9-20.

Bishop, Alan (2000): “Enseñanza de las matemáticas: ¿Cómo beneficiar a todos los alumnos?” en María Núria Gorgorió, Jordi Deulofeu y Alan Bishop (coords.): Matemáticas y educación. Barcelona: Editorial Graó.

Bolívar, Antonio y López, Lourdes (2009): "Las grandes cifras del fracaso y los riesgos de exclusión educativa". Profesorado. Revista de currículum y formación de profesorado, 13, 52-78.

Blázquez, Carola; Álvarez, Pamela; Bronfman, Nicolás y Espinosa, Juan F. (2009): "Factores que influencian la motivación de escolares por las áreas tecnológicas e ingeniería". Calidad en la Educación, 31, 46-64. DOI: https://doi.org/10.31619/caledu.n31.162.

Brandell, Gerd y Staberg, Else-Marie (2008): "Mathematics: A Female, Male or Gender-Neutral Domain? A Study of Attitudes Among Students at Secondary Level”. Gender and Education, 20, 495-509. DOI: https://doi.org/10.1080/09540250701805771.

Bueno, Ángeles (2006): “Actitudes del profesorado ante la educación de alumnos de altas capacidades”. Faisca. Revista de altas capacidades, 11 (13), 76-100.

Bull, Rebecca y Scerif, Gaia (2001): “Executive Functioning as a Predictor of Children's Mathematics Ability: Inhibition, Switching, and Working Memory". Developmental Neuropsychology, 19 (3), 273-293. DOI: https://doi.org/10.1207/S15326942DN1903_3.

Cadenas, Cristina y Huertas, Francisco Javier (2013): “Informe PISA en España. Un análisis al detalle”. Profesorado. Revista de currículum y formación del profesorado, 17 (2), 243-262.

Calvo, Guadalupe (2018): "Las identidades de género según las y los adolescentes. Percepciones, desigualdades y necesidades educativas". Contextos educativos: Revista de educación, 21. DOI: https://doi. org $/ 10.18172 / \operatorname{con} .3311$. 
Campos, C. (2006). Actitud hacia las matemáticas: diferencias de género entre estudiantes de sexto de primaria y tercer grado de secundaria. Tesis inédita de Maestría. México. Cinvestav-ipn.

Cantoral, Ricardo; Reyes-Gasperini, Daniela y Montiel, Gisela (2014): “Socioepistemología, matemáticas y realidad”. Revista Latinoamericana de Etnomatemática, 7 (3), 91-116. Recuperado de: https://www.redalyc.org/articulo.oa?id=274032530006.

Carey, Emma; Hill, Francesca; Devine, Amy y Szücs, Dénes (2016): “The Chicken or the Egg? The Direction of the Relationship Between Mathematics Anxiety and Mathematics Performance". Frontiers in Psychology, 6, 23-42. DOI: https://doi.org/10.3389/fpsyg.2015.01987.

Celis, G.A. (1986). Los subtests de razonamiento abstracto, razonamiento verbal y relaciones espaciales del D.A.T., como elementos predictivos de rendimiento académico en la U.I.A.Tesis de Licenciatura en Psicología. México. Universidad Iberoamericana.

Chamorro-Premuzic, Tomas y Furnham, Adrian (2008): "Personality, Intelligence and Approaches to Learning as Predictors of Academic Performance". Personality and individual differences, 44, 1596-1603. DOI: https://doi.org/10.1016/j.paid.2008.01.003.

Chamorro-Premuzic, Tomas; Quiroga, María Ángeles y Colom, Roberto (2009): “Intellectual Competence and Academic Performance: A Spanish Study". Learning and Individual Differences, 19, 486- 491. DOI: https://doi.org/10.1016/j.lindif.2009.05.002.

Consorcio PISA. (2004). Aprendizaje para el mañana, Primeros resultados PIS A 2003. Recuperado de: https:// www.oecd.org/pisa/39732493.pdf.

Coyne, Sarah M.; Linder, Jennifer Ruh; Rasmussen, Eric E.; Nelson, David A. y Collier, Kevin M. (2014): “It's a Bird! It's a Plane! It's a Gender Stereotype!: Longitudinal Associations Between Superhero Viewing and Gender Stereotyped Play". Sex Roles, 9-10 (70), 416-430. DOI: https://doi.org/10.1007/ s11199-014-0374-8.

Del Río, María Francisca; Strasser, Katherine y Susperreguy, María Inés (2016): “¿Son las habilidades matemáticas un asunto de Género? Los estereotipos de género acerca de las matemáticas en niños y niñas de Kínder, sus familias y educadoras". Calidad en la educación, 45, 20-53. Recuperado de: https:// www.cned.cl/sites/default/files/cse_articulo1221.pdf.pdf.

Desco, Manuel; Navas-Sánchez, Francisco J.; Sánchez-González, Javier; Reig, Santiago; Robles, Olalla; Franco, Carolina; Guzmán-De-Villoria, Juan A.; Gracía-Barreano, Pedro y Arango, Celso (2011): "Mathematically Gifted Adolescents Use More Extensive and More Bilateral Areas of the Fronto-Parietal Network than Controls During Executive Functioning and fluid Reasoning Tasks". NeuroImage, 57, 281-292. DOI: https://doi.org/10.1016/j.neuroimage.2011.03.063.

Dowker, Ann; Sarkar, Amar y Yen Looi, Chung (2016): "Mathematics Anxiety: ¿What Have We Learned in 60 Years?”. Frontiers in Psychology, 7, 508. DOI: https://doi.org/10.3389/fpsyg.2016.00508.

Edel, Rubén (2003): “El rendimiento académico: concepto, investigación y desarrollo". REICE - Revista Electrónica Iberoamericana sobre Calidad, Eficacia y Cambio en Educación, 1 (2). Recuperado del: https:// www.redalyc.org/pdf/551/55110208.pdf. 
Else-Quest, Nicole M.; Hyde, Janet Shibley y Linn, Marcia C. (2010): “Cross-National Patterns of Gender Differences in Mathematics: A Meta-Analysis”. Psychological Bulletin, 136, 103-127. DOI: https:// doi.org/10.1037/a0018053.

Eudave, Daniel (1994): "Las actitudes hacia las matemáticas de los maestros y alumnos de bachillerato". Educación Matemática, 6 (1), 46-58. Recuperado de: http:// funes.uniandes.edu.co/9693/.

Farfán, Rosa María y Simón, Guadalupe (2017): “Género y matemáticas: una investigación con niñas y niños talento”. Acta Scientiae, 19 (3), 427- 446.

Forgasz, Helen J. (2000): “The Gender-Stereotyping of Mathematics: Pre-Service Teachers' Views”. Comunicacion presentada en la Annual Conference of the Australian Association for Research in Education, Sydney. Recuperado de: https://www.aare.edu.au/data/publications/2000/for00168.pdf.

Frenzel, Anne C.; Pekrun, Reinhard y Goetz, Thomas (2007): “Girls and Mathematics - A 'Hopeless' Issue ? A Control-Value Approach to Gender Differences in Emotions Towards Mathematics”. European Journal of Psychology of Education, 22, 497-514. DOI: https://doi.org/10.1007/bf03173468.

Garbanzo Vargas, Guiselle María (2007): "Factores asociados al rendimiento académico en estudiantes universitarios, una reflexión desde la calidad de la educación superior pública”. Revista Educación, 31, 43- 63. Recuperado de: https://www.redalyc.org/articulo.oa?id=44031103.

García de León, María Antonia (2004). Élites discriminadas (sobre el poder de las mujeres). Colombia: Editorial Anthopodos.

Gasco-Txabarri, Javier (2017): "Diferencias en el uso de estrategias en el aprendizaje de las matemáticas en enseñanza secundaria según el sexo”. Cuadernos de Investigación Educativa, 8(1), 47-59. DOI: https:/ / doi.org/10.18861/cied.2017.8.1.2638.

Gil, Núria; Blanco, Lorenzo J. y Guerrero, Eloísa (2005): "El dominio afectivo en el aprendizaje de las Matemáticas. Una revisión de sus descriptores básicos". Revista iberoamericana de educación matemática, 2, 15-33. Recuperado de: http://www.fisem.org/www/union/revistas/2005/2/Union_002_004.pdf.

Gómez Chacón, Inés María (2000). Matemática emocional. Madrid: Narcea.

Gómez Chacón, Inés María (2001): “The Emotional Dimesion in Mathematics Education: A Bibliography". Statistical Education Research Newsletter, 2 (2). International Association for Statistical Education. Recuperado de: https://iase-web.org/documents/SERJ/Newsmay01.pdf.

González-Pienda, Julio Antonio; Fernández-Cueli, Marisol; García, Trinidad; Suárez, Natalia; Fernández, Estrella; Tuero-Herrero, Elián y da Silva, Elza Helene (2012). "Diferencias de género en actitudes hacia las matemáticas en la enseñanza obligatoria”. Revista iberoamericana de psicología y salud, 3 (1), 55-73. Recuperado de: http:/ /www.redalyc.org/articulo.oa?id=245122736004.

Gunderson, Elizabeth; Ramírez, Gerardo; Levine, Susan C. y Beilock, Sian L. (2011): “The Role of Parents and Teachers in the Development of Gender-Related Math Attitudes”. Sex Roles, 3-4 (66), 153166. DOI: https://doi.org/10.1007/s11199-011-9996-2.

Hanna, Gila (2003): “Reaching Gender Equity in Mathematics Education”. The Educational Forum, 67 (3), 204-214. DOI: https://doi.org/10.1080/00131720309335034. 
Herbert, Jennifer y Stipek, Deborah (2005): “The Emergence of Gender Differences in Children's Perceptions of their Academic Competence". Journal of Applied Developmental Psychology: An International Lifespan Journal, 3 (26), 276- 295. DOI: https://doi.org/10.1016/j.appdev.2005.02.007.

Inda-Caro, Mercedes; Rodríguez-Menéndez, Carmen y Peña-Calvo, Vicente (2010): "PISA 2006: la influencia del género en los conocimientos y competencias científicas". Revista Iberoamericana de Educación, 51 (2), 1-12. Recuperado de: https://www.ugr.es/ fjjrios/pce/media/4-5-c-PisaGenero.pdf.

Kloosterman, Peter; Hagemeyer, Janet; Ponniah, Ann G. y Essex, N. Kathryn (2001): "Mathematics as a Gendered Domain in the United States". The American Educational Research Association, 13, 1-15. Recuperado de: http://citeseerx.ist.psu.edu/viewdoc/download?doi=10.1.1.22.5640\&rep=rep1\&type $=$ pdf.

Kurtz-Costes, Beth; Rowley, Stephanie J.; Harris-Britt, April y Woods, Taniesha A. (2008): “Gender Stereotypes About Mathematics and Science and Self-perceptions of Ability in Late Childhood and Early Adolescence”. Merrill Palmer Quarterly, 3 (54), 386-409. DOI: https://doi.org/10.1353/mpq.0.0001.

Hwa Lee, Kyeong y Sriraman, Bharath (2012): "Gifted Girls and Non-Mathematical Aspirations: A Longitudinal Case Study of Two Gifted Korean Girls”. Gifted Child Quaterly, 56 (1), 3-14. DOI: https:/ / doi.org/10.1177/0016986211426899.

Llanos, Viviana Carolina y Otero, María Rita (2015): “La incidencia de las funciones didácticas topogénesis, mesogénesis y cronogénesis en un recorrido de estudio y de investigación: el caso de las funciones polinómicas de segundo grado". Revista Latinoamericana de Investigación en Matemática Educativa (Relime), 8 (2), 245-275. Recuperado de: http://www.scielo.org.mx/pdf/relime/v18n2/v18n2a5.pdf.

Markova, Dawna y Powell, Anne (1997). Cómo desarrollar la inteligencia de sus hijos. México. Selector.

Marsh, Herbert W. y Martin, Andrew J. (2010): “Academic Self-Concept and Academic Achievement: Relations and Causal Ordering". British Journal of Educational Psychology, 81, 59-77. DOI: https://doi. org/10.1348/000709910X503501.

López Martínez, María.; Reverte, Ginés Marco y Palacios Manzano, Mª Mercedes (2016): “El fracaso escolar en España y sus regiones: Disparidades territoriales". Revista de estudios regionales, 107, 121 155. Recuperado de: http://www.revistaestudiosregionales.com/documentos/articulos/pdf-articulo-2506.pdf.

Mingo, Araceli (2006). ¿Quién Mordió la manzana? Sexo, origen socialy desempeño en launiversidad. México. Fondo de Cultura Económica.

Ministerio de educación, cultura y deporte (2001). Proyecto para la evaluación internacional de los alumnos. Proyecto PIS A. Recuperado de: https://www.educacionyfp.gob.es/inee/dam/jcr:39ae75ee-8fa0-4af9-b24ad8b435d806d4/pisa2000-int.pdf.

Ministerio de educación, cultura y deporte (2004). El aprendizaje para el Mundo del mañana. Primeros resultados del Programa PIS A 2003. Recuperado de: https://www.educacionyfp.gob.es/inee/dam/jcr:2ca96736bc2c-48a8-83c7-7eb18da066c5/pisa2003resumenocde.pdf.

Ministerio de educación, cultura y deporte (2005). Resultados en España del estudio PISA 2000. Recuperado de: https://www.educacionyfp.gob.es/inee/dam/jcr:eb6fde04-a7c1-465a-88b4-e51465383807/ pisa2000infnacional.pdf. 
Ministerio de educación, cultura y deporte (2014). PIS A 2012. Programa para la Evaluación Internacional de los Alumnos. Informe español. Recuperado de: https://www.educacionyfp.gob.es/inee/dam/jcr:99d0104f37ec-4dc7-b6d7-0fc6fddbef7c/presentacionpisa2012.pdf.

Ministerio de educación, cultura y deporte (2016). PIS A 2015. Programa para la Evaluación Internacional de los Alumnos. Informe español. Recuperado de: https://www.educacionyfp.gob.es/inee/dam/jcr:e4224d22f7ac-41ff-a0cf-876ee5d9114f/pisa2015preliminarok.pdf.

Ministerio de la presidencia, relaciones con las cortes e igualdad (2019). Instituto de la mujer y para la igualdad de oportunidades. España. Recuperado de: http://www.inmujer.gob.es/actualidad/noticias/2019/ ingenieraunivers.htm.

Mizala, Alejandra; Martínez, Francisco y Martínez, Salomé (2015): “Pre-Service Elementary School Teachers' Expectations About Student Performance: How their Beliefs are Affected by their Mathematics Anxiety and Student's Gender". Teaching and Teacher Education, 50, 70-78. DOI: https://doi. org/10.1016/j.tate.2015.04.006.

Morales, Cesáreo (1998): “Actitudes de los escolares hacia la computadora y los medios para el aprendizaje”. Tecnología y Comunicación Educativa, 28, 51-65.

Muelas, Álvaro (2013): "Influencia de la variable de personalidad en el rendimiento académico de los estudiantes cuando finalizan la Educación Secundaria Obligatoria (ESO) y comienzan Bachillerato". Historia y Comunicación Social, 18, 115-126. DOI: https://doi.org/10.5209/rev_HICS.2013.v18.44230.

Muelas, Álvaro y Beltrán, Jesús A. (2011): "Variables influyentes en el rendimiento académico de los estudiantes”. Revista de Psicología y Educación, 6, 173-196. Recuperado de: http://www.revistadepsicologiayeducacion.es/pdf/65.pdf.

Nasiriyan, A. Azar; H.K. Noruzy, A. y Dalvand, M.R. (2011): “A Model of Self-Efficacy, Task Value, Achievement Goals, Effort and Mathematics Achievement". International Journal of Academic Research, $3(2), 612-618$.

Nosek, Brian A.; Smyth, Frederick L.; Sriram, N.; Lindner, Nicole M.; Devos, Thierry; Ayala, Alfonso; Bar-Anan, Yoav; Bergh, Robin; Cai, Huajian; Gonsalkorale, Karen; Kesebir, Selin; Maliszewski, Norbert; Neto, Félix; Olli, Eero; Park, Jaihyun; Schnabel, Konrad; Shiomura, Kimihiro; Tulbure, Bogdan Tudor; Wiers, Reinout W.; Somogyi, Mónika; Akrami, Nazar; Ekehammar, Bo; Vianello, Michelangelo; Banaji, Mahzarin R. y Greenwald, Anthony G. (2009): "National Differences in Gender-Science Stereotypes Predict National Sex Differences in Science and Math Achievement". PNAS Proceedings of the National Academy of Sciences of the United States of America, 106 (26), 10593-10597. DOI: https://doi. org/10.1073/pnas.0809921106.

OECD (2007). PISA 2006: Science Competencies for Tomorrow's World Executive. Recuperado de: https:// www.google.com $/$ url? sa $=$ t\&rct $=j \& q=\& e s r c=s \&$ source $=$ web\&cd $=3 \& v e d=2 a h U K E w j S 8 M 6 D-$

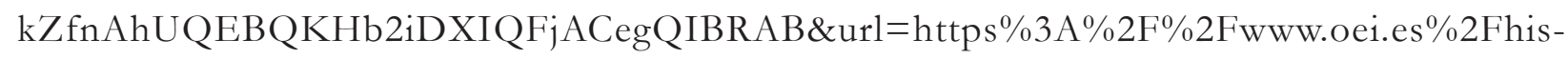
torico $\% 2$ Fevaluacioneducativa $\% 2$ FInformePISA2006-FINALingles.pdf\&usg=AOvVaw1x1RIcvh4qyz7AF7BTPv_w.

OECD (2010). PISA 2009 Results: Executive Summary. Recuperado de: https://www.oecd.org/pisa/pisaproducts/46619703.pdf. 
Pelegrina, Santiago; García Linares, María Cruz y Casanova, Pedro Félix (2002): "Parenting Styles and Adolescents' Academic Performance”. Infancia y Aprendizaje, 25 (2), 147-168. DOI: https://doi. org/10.1174/021037002317417796.

Pérez, Luz F.; González, C. y Beltrán, Jesús A. (2009): “Atención, inteligencia y rendimiento académico”. Revista de Psicología y Educación, 1 (4), 57-72.

Preckel, Franzis; Götz, Thomas; Pekrun, Reinhard (2008): “Gender Differences in Gifted and Average Ability Students: Comparing Girls and Boys Achievement, Self-concept, Interest and Motivation in Mathematics". Gifted Child Quarterly, 52, 146-159. DOI: https://doi.org/10.1177/0016986208315834.

Ramírez, M. (2006). Influencia de la visión de género de las docentes en las interacciones que establecen con el alumnado en las clases de matemáticas. Tesis inédita de Maestría. México. Cinvestav-ipn

Rodríguez, Carmen; Delgado, Purificación y Bakieva, Margarita (2011): “Actividades extraescolares y rendimiento académico: diferencias en autoconcepto y género". Revista de Investigación Educativa, 29 (2), 447-465. Recuperado de: https://dialnet.unirioja.es/servlet/articulo?codigo=3673766.

Rodríguez, Daniel y Guzmán, Remedios (2018): "Relación entre perfil motivacional y rendimiento académico en Educación Secundaria Obligatoria”. Estudios sobre educación, 34, 199-217. DOI: https:/ / doi.org/10.15581/004.34.199-217.

Rodríguez-Mantilla, Jesús Miguel; Fernández-Díaz, Ma José y Jover, Gonzalo (2018): "PISA 2015: Predictores del rendimiento en Ciencias en España". Revista de Educación, 38, 75-102. DOI: https://doi. org/10.4438/1988-592X-RE-2017-380-373.

Ruiz de Miguel, Covadonga (2009): "Las escuelas eficaces: un estudio multinivel de factores explicativos del rendimiento escolar en el área de matemáticas”. Revista de educación, 348, 355-376. Recuperado de: https://dialnet.unirioja.es/servlet/articulo?codigo $=2801304$.

Schaffer, Rudolph (2000). Desarrollo social. México: Siglo XXI editores

Schillinger, Frieder L.; Vogel, Stephan E.; Diedrich, Jennifer; Grabner, Roland H. (2018): "Math Anxiety, Intelligence, and Performance in Mathematics: Insights from the German Adaptation of the Abbreviated Math Anxiety Scale (AMAS-G)". Learning and Individual Differences, 61, 109-119. DOI: https:// doi.org/10.1016/j.lindif.2017.11.014.

Song, Lynda Jiwen; Huang, Guo-hua; Peng, Kelly Z.; Law, Kenneth S.; Wong, Chi-Sum, Chen, Zhijun (2010): “The Differential Effects of General Mental Ability and Emotional Intelligence on Academic Performance and Social Interactions”. Intelligence, 38, 137-143. DOI: https://doi.org/10.1016/j.intell.2009.09.003.

Takayama, Keita (2008): “The Politics of International League Tables: PISA in Japan’s Achievement Crisis Debate". Comparative Education, 44 (4), 387-407. DOI: https://doi.org/10.1080/03050060802481413.

Ursini, Sonia (2009). Aspectos educativos y género en el aprendizaje de las matemáticas en escuelas secundarias del Distrito Federal. Cuaderno de Trabajo 15. México: Instituto Nacional de las Mujeres.

Ursini, Sonia (2010): "Diferencias de género en la representación social de las matemáticas: un estudio con alumnos de secundaria". Investigación feminista, epistemología, metodología y representaciones social- 
es, 379-398. Recuperado de: http://biblioteca.clacso.edu.ar/Mexico/ceiich-unam/20170428032751/ pdf_1307.pdf.

Ursini, Sonia (2013): "Las diferencias de género en matemáticas: una realidad poco atendida desde las representaciones sociales" en Fátima Flores-Palacios (ed.): Representaciones sociales y contextos de investigación con perspectiva de género. México: Universidad Nacional Autónoma de México.

Ursini Sonia; Ramírez-Mercado, Martha P.; y Sánchez, Gabriel (2007): “Using Technology in the Mathematics Class: How this Affects Students' Achievement and Attitudes. Proceedings of the $8^{\text {th }}$ ICTMT, (Integration of ICT into Learning Processes)" (CD-ROM). República checa: University of Hradec Králové.

Ursini, Sonia y Ramírez-Mercado, Martha P. (2017). "Equidad, género y matemáticas en la escuela mexicana”. Revista Colombiana de Educación, (73), 213-234. Recuperado de: http://www.scielo.org.co/pdf/ rcde/n73/0120-3916-rcde-73-00213.pdf.

Ursini, Sonia y Sánchez, Gabriel (2008): “Gender, Technology and Attitude Towards Mathematics: A Comparative Longitudinal Study with Mexican Students”. ZDM, Mathematics Education, 40 (4), 559577. DOI: https://doi.org/10.1007/s11858-008-0120-1.

Ursini, Sonia; Sánchez, Gabriel; Orendain, Mónica y Butto, Cristianne (2004): “El uso de la tecnología en el aula de matemáticas: diferencias de género desde la perspectiva de los docentes”. Enseñanza de las Ciencias, 22 (3), 409-424.

Valero, Paola; García, Gloria; Salazar, Claudia; Mancera, Gabrial; Camelo, Francisco y Romero, Julio (2013). Procesos de inclusión/exclusión: Subjetividades en educación matemática elemental. Bogotá: Universidad Pedagógica Nacional; Universidad de Aalborg; Universidad Distrital Francisco José de Caldas; Colciencias.

Zuaza, Enrique y Rodríguez, Roberto (2002): “Enseñar y aprender Matemáticas”. Revista de Educación, 329, 239-256. Recuperado de: https://www.researchgate.net/publication/39208058_Ensenar_y_ aprender_matematicas.

\section{Notas biográficas}

Silvia Fuentes De Frutos es Doctorada en Psicología por la URV (Universidad Rovira i Virgili), trabaja actualmente como Profesora asociada en el Departamento de Educación de la UNIR (Universidad Internacional de la Rioja). Está acreditada por la ANECA como Profesora Ayudante Doctor, posgraduada en Gestión de Recursos Humanos y licenciada en Psicología. Ha sido profesora en el Grado de Psicología, Psicopedagogía y Magisterio en la URV, profesora en el Grado de Aviación en CESDA (Centro de Estudios Superiores sobre la Aviación) y profesora en el Grado de Marketing en ESERP Business School. Además ha desarrollado su experiencia docente en diferentes Universidades en Francia. Directora de TFG y TFM, Su especialidad se centra en la Psicosociología, la Psicología Social y Psicología de la Educación. Actualmente forma parte del Proyecto de Innovación Docente CoLE (Collaborative Learning Environment) de la Agencia de Gestión de Ayudas Universitarias y de Investigación (AGAUR). 
Víctor Renobell Santaren es Doctor en Sociología. Profesor acreditado (ANECA). Actualmente profesor y Coordinador del grado de Ciencias Políticas de la UNIR (Universidad Internacional de La Rioja). Ha sido profesor en la UCJC (Universidad Camilo José Cela) en Educación y Máster de Secundaria, en ESERP (Barcelona) en las licenciaturas de ADE, Comunicación y Marketing, en EUNCET Business School (Centro Adscrito a la Universidad Politécnica de Catalunya) en ADE y Marketing, y en el TECNOCAMPUS (Escola Universitaria del Maresme Centro Adscrito a la Universidad Pompeu Fabra) en ADE, Marketing y Turismo. He sido profesor y director del área de comunicación y sociedad de la Universitat Oberta de Catalunya, Director Académico del Máster en Procesos editoriales y de Comunicación de la UOC, Manager en la editorial Edebé, y Director de la delegación de Bureau Veritas Business School de Barcelona. Director de TFG, TFM y Tesis Doctorales. 